International Journal of Pure and Applied Mathematics

Volume 82 No. 4 2013, 631-637

ISSN: 1311-8080 (printed version); ISSN: 1314-3395 (on-line version)

url: http://www.ijpam.eu

doi: http://dx.doi.org/10.12732/ijpam.v82i4.12

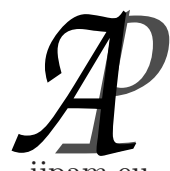

ijpam.eu

\title{
DECOMPOSITION ALGORITHMS POTENTIALS FOR THE NON-HOMOGENEOUS GENERALIZED NETWORKED PROBLEMS OF LINEAR-FRACTIONAL PROGRAMMING
}

\author{
L.A. Pilipchuk \\ Belarussian State University \\ Nezalezhnosti Ave. 4, 220050, Minsk, BELARUS
}

\begin{abstract}
We use potentials for calculate a reduced costs in the increment of the objective function for the linear-fractional non-homogeneous flow programming optimization problem with additional constraints of general kind. The effective algorithm for solution of the system of potentials for a sparse matrix is considered.
\end{abstract}

AMS Subject Classification: 65K05, 90C08, 90C35, 05C50, 15A03, 15A06 Key Words: linear-fractional programming, two-component sparse matrix, increment of the objective function, decomposition, potentials, generalized multinetwork

\section{Introduction}

We consider the linear-fractional non-homogeneous flow programming optimization problem with additional constraints of general kind:

$$
f(x)=\frac{p(x)}{q(x)}=\frac{\sum_{(i, j) \in U} \sum_{k \in K(i, j)} p_{i j}^{k} x_{i j}^{k}+\beta}{\sum_{(i, j) \in U} \sum_{k \in K(i, j)} q_{i j}^{k} x_{i j}^{k}+\gamma} \longrightarrow \max ,
$$

Received: November 1, 2012

(c) 2013 Academic Publications, Ltd. url: www.acadpubl.eu 


$$
\begin{gathered}
\sum_{j \in I_{i}^{+}\left(U^{k}\right)} x_{i j}^{k}-\sum_{j \in I_{i}^{-}\left(U^{k}\right)} \mu_{j i}^{k} x_{j i}^{k}=a_{i}^{k}, i \in I^{k}, k \in K \\
\sum_{(i, j) \in U} \sum_{k \in K(i, j)} \lambda_{i j}^{k p} x_{i j}^{k}=\alpha_{p}, p=\overline{1, l} ; \\
\sum_{k \in K_{0}(i, j)} x_{i j}^{k} \leq d_{i j}^{0}, x_{i j}^{k} \geq 0, k \in K_{0}(i, j),(i, j) \in U_{0} ; \\
0 \leq x_{i j}^{k} \leq d_{i j}^{k}, k \in K_{1}(i, j),(i, j) \in U ; \\
x_{i j}^{k} \geq 0, k \in K(i, j) \backslash K_{1}(i, j),(i, j) \in U \backslash U_{0},
\end{gathered}
$$

where $G=(I, U)$ is a finite oriented connected multigraph (multinetwork) without multiple arcs and loops, $I$ is a set of nodes and $U \subset I \times I$ is a set of multiarcs. The finite non-empty set $K=\{1, \ldots,|K|\}$ is the set of different products (commodities) transported through the multinetwork $G$. Let us denote a connected network corresponding to a certain type of flow $k \in K: G^{k}=\left(I^{k}, U^{k}\right), I^{k} \subseteq I$, $U^{k}=\left\{(i, j)^{k}:(i, j) \in \widehat{U}^{k}\right\}, \widehat{U}^{k} \subseteq U-$ a set of arcs of the multinetwork $G$ carrying the flow of type $k \in K, I^{k}=I\left(U^{k}\right), I\left(U^{k}\right)=\left\{i \in I: i \in I^{k}\right\}$ is the set of nodes used for transporting (producing/consuming/transiting) the $k^{\text {th }}$ product. In order to distinguish the products, which can simultaneously pass through an multiarc $(i, j) \in U$, we introduce the set $K(i, j)=\left\{k \in K:(i, j)^{k} \in U^{k}\right\}$. Similarly, $K(i)=\left\{k \in K: i \in I^{k}\right\}$ is the set of products simultaneously transported through a node $i \in I$. Let's define a set $U_{0}$ as an arbitrary subset of multiarcs of the multinetwork $G, U_{0} \subseteq U$. Each multiarc $(i, j) \in U_{0}$ has an aggregate capacity constraint for a total amount of transported products from a subset $K_{0}(i, j) \subseteq K(i, j),\left|K_{0}(i, j)\right|>1$. For all multiarcs $(i, j) \in U$ we assume the amount of each product $k \in K(i, j)$ to be non-negative. For a set $K_{1}(i, j)$ are true the following conditions: $K_{1}(i, j)=K(i, j) \backslash K_{0}(i, j)$, if $(i, j) \in U_{0}$ and $K_{1}(i, j) \subseteq K(i, j)$, if $(i, j) \in U \backslash U_{0}$. Moreover, each multiarc $(i, j) \in U$ can be equipped with carrying capacities for products from a set $K_{1}(i, j)$, where $K_{1}(i, j) \subseteq K(i, j)$ is an arbitrary subset of products transported through the multiarc $(i, j) . I_{i}^{+}\left(U^{k}\right)=\left\{j \in I^{k}:(i, j) \in U^{k}\right\}, I_{i}^{-}\left(U^{k}\right)=\left\{j \in I^{k}:(j, i) \in\right.$ $\left.U^{k}\right\} ; x_{i j}^{k}$ - amount of the $k^{\text {th }}$ product transported through an multiarc $(i, j)$; $d_{i j}^{k}$ - carrying capacity of an multiarc $(i, j)$ for the $k^{\text {th }}$ product; $d_{i j}^{0}$ - aggregate capacity of an multiarc $(i, j) \in U_{0}$ for a total amount of products $K_{0}(i, j)$; $\lambda_{i j}^{k p}$ - weight of a unit of the $k^{\text {th }}$ product transported through an multiarc $(i, j)$ in the $p^{\text {th }}$ additional constraint; $\mu_{i j}^{k}-$ a flow transformation coefficient for $\left.\left.\operatorname{arc}(i, j)^{k}, \quad \mu_{i j}^{k} \in\right] 0,1\right] ; \alpha_{p}$ - total weighted amount of products imposed by 
the $p^{\text {th }}$ additional constraint; $a_{i}^{k}$ - intensity of a node $i$ for the $k^{\text {th }}$ product, $p_{i j}^{k}, q_{i j}^{k}, \beta, \gamma \in \mathbf{R}$.

\section{Sparse Systems for a Potentials}

The formula of the increment of the objective function (1) for the extreme linear-fractional non-homogeneous problem of flow programming (1)-(6) with additional constraints has the following kind:

$$
\Delta f=\frac{\sum_{k \in K} \sum_{(\tau, \rho)^{k} \in U_{N}^{k}} \widetilde{\Delta}^{k}(\tau, \rho) \Delta x_{\tau \rho}^{k}+\sum_{(i, j) \in U^{*}} r_{i j} \Delta z_{i j}}{\sum_{k \in K} \sum_{(\tau, \rho)^{k} \in U^{k} \backslash U_{L}^{k}} \Delta_{Q}^{k}(\tau, \rho)\left(x_{\tau \rho}^{k}+\Delta x_{\tau \rho}^{k}\right)+Q+\gamma},
$$

where

$$
\begin{gathered}
\widetilde{\Delta}^{k}(\tau, \rho)=\Delta^{k}(\tau, \rho)-\sum_{p=1}^{l} r_{p} \Lambda_{\tau \rho}^{k p}-\sum_{(i, j) \in U^{*}} r_{i j} \delta_{i j}\left(B_{\tau \rho}^{k}\right), \\
\Delta^{k}(\tau, \rho)=\Delta_{P}^{k}(\tau, \rho)-f(x) \Delta_{Q}^{k}(\tau, \rho), \\
\Delta_{P}^{k}(\tau, \rho)=p_{\tau \rho}^{k}+\sum_{(i, j)^{k} \in U_{L}^{k}} p_{i j}^{k} \delta_{i j}^{k}(\tau, \rho), \\
\Delta_{Q}^{k}(\tau, \rho)=q_{\tau \rho}^{k}+\sum_{(i, j)^{k} \in U_{L}^{k}} q_{i j}^{k} \delta_{i j}^{k}(\tau, \rho), \\
\Lambda_{\tau \rho}^{k p}=\lambda_{\tau \rho}^{k p}+\sum_{(i, j)^{k} \in U_{L}^{k}} \lambda_{i j}^{k p} \delta_{i j}^{k}(\tau, \rho),(\tau, \rho)^{k} \in U^{k} \backslash U_{L}^{k}, p=\overline{1, l}, \\
\delta_{i j}\left(B_{\tau \rho}^{k}\right)=\left\{\begin{array}{c}
\delta_{i j}^{k}(\tau, \rho), k \in K_{0}(i, j), \\
(i, j) \in U_{0},(\tau, \rho)^{k} \in U^{k} \backslash U_{L}^{k}, k \in K .
\end{array}\right. \\
r_{p}=\sum_{k \in K} \sum_{(\tau, \rho)^{k} \in U_{B}^{k}} \Delta^{k}(\tau, \rho) \nu_{t(\tau, \rho)^{k}, p}, p=\overline{1, l}, \\
r_{i j}=\sum_{k \in K} \sum_{(\tau, \rho)^{k} \in U_{B}^{k}} \Delta^{k}(\tau, \rho) \nu_{t(\tau, \rho)^{k}, l+\xi(i, j)},(i, j),(i, j) \in U^{*},
\end{gathered}
$$

where $x=\left(x_{i j}^{k},(i, j) \in U, k \in K(i, j)\right)$ be a multiflow of the problem (1)-(6) i. e. components of the vector $x$ meet the conditions (2)-(6). Along with the 
multiflow $x$ let us define support multiflow $\left\{x, U_{S}\right\}$ as a pair [1], containing of an arbitrary multiflow $x$ and a support $[1,4] U_{S}$ of multigraph $G=\{I, U\}$ of the problem (1)-(6), $U_{S}=\left\{U_{S}^{k}, k \in K, U^{*}\right\}, U_{S}^{k} \subset U^{k}, k \in K ; U^{*} \subseteq \bar{U}_{0}, \bar{U}_{0}=$ $\left\{(i, j) \in U_{0}:\left|K_{S}^{0}(i, j)\right|>1\right\}, K_{S}(i, j)=\left\{k \in K(i, j):(i, j)^{k} \in U_{S}^{k}\right\},(i, j) \in U$, $K_{S}^{0}(i, j)=K_{S}(i, j) \bigcap K_{0}(i, j),(i, j) \in U_{0}$ of the problem (1)-(6). A support $U_{S}$ of multigraph $G=\{I, U\}$ of the problem (1)-(6) includes a support $U_{L}=$ $\left\{U_{L}^{k}, k \in K\right\}$ for system (2) and the set $U_{B}=\left\{U_{B}^{k}, k \in K\right\}$ of bicycling arcs [1]-[4].

Let's consider some other multiflow

$$
\bar{x}=\left(\bar{x}_{i j}^{k}=x_{i j}^{k}+\Delta x_{i j}^{k}:(i, j) \in U, k \in K(i, j)\right)
$$

Then $\Delta x=\left(\Delta x_{i j}^{k},(i, j) \in U, k \in K(i, j)\right)$ is the vector of flow increments along the multiarc $(i, j) \in U$,

$$
\begin{gathered}
z_{i j}=\sum_{k \in K_{0}(i, j)} x_{i j}^{k}, \quad \bar{z}_{i j}=\sum_{k \in K_{0}(i, j)} \bar{x}_{i j}^{k}, \\
\Delta z_{i j}=\bar{z}_{i j}-z_{i j}=\sum_{k \in K_{0}(i, j)} \Delta x_{i j}^{k}, \quad(i, j) \in U_{0},
\end{gathered}
$$

$\delta^{k}(\tau, \rho)=\left(\delta_{i j}^{k}(\tau, \rho), \quad(i, j)^{k} \in U^{k}\right)$ - characteristic vector, entailed by arc $(\tau, \rho)^{k} \in U^{k} \backslash U_{L}^{k}$ concerning a support $U_{L}^{k}$ for system (2), $k \in K[2]$,

$$
Q=\sum_{k \in K} \sum_{(i, j)^{k} \in U_{L}^{k}} q_{i j}^{k}\left(\widetilde{x}_{i j}^{k}-\sum_{(\tau, \rho)^{k} \in U^{k} \backslash U_{L}^{k}} \widetilde{x}_{\tau \rho}^{k} \delta_{i j}^{k}(\tau, \rho)\right),
$$

where $\tilde{x}^{k}=\left(\tilde{x}_{i j}^{k},(i, j)^{k} \in U^{k}\right)$ - is partial solution of the nonhomogeneous system $(2)$ and $\delta^{k}(\tau, \rho)=\left(\delta_{i j}^{k}(\tau, \rho),(i, j)^{k} \in U^{k}\right),(\tau, \rho)^{k} \in U^{k} \backslash U_{L}^{k}, k \in K$ is the system of characteristic vectors, entailed by an $\operatorname{arc}(\tau, \rho)^{k} \in U^{k} \backslash U_{L}^{k}, k \in K$ for the fixed $k \in K[2,4]$.

Remark. We use the partial solution

$$
\widetilde{x}^{k}=\left(\widetilde{x}_{i j}^{k},(i, j)^{k} \in U^{k}\right), k \in K
$$

which is constructed to the following rules: non-supporting elements $(\tau, \rho)^{k} \in$ $U^{k} \backslash U_{L}^{k}, k \in K$ are equal to zeros and supporting elements $(i, j)^{k} \in U_{L}^{k}, k \in K$ satisfy system (2). 
For calculation of reduced costs (8) we will use the sparse system potentials. Let's write down system (11)-(13) of potentials $r, \quad u^{k}, k \in K$ :

$$
\begin{gathered}
r=\left(r_{p}: p=\overline{1, l} ; r_{i j},(i, j) \in U^{*}\right), \\
u^{k}=\left(u_{i}^{k}, i \in I^{k}\right), k \in K,
\end{gathered}
$$

for a support $U_{S}[5]$ of the multigraph $G$ for a problem (1)-(6):

$$
\begin{gathered}
u_{i}^{k}-\mu_{i j}^{k} u_{j}^{k}+\sum_{p=1}^{l} \lambda_{i j}^{k p} r_{p}=-\left(p_{i j}^{k}-f(x) q_{i j}^{k}\right), \\
(i, j) \in U \backslash U^{*}, k \in K_{S}(i, j) ; \\
u_{i}^{k}-\mu_{i j}^{k} u_{j}^{k}+\sum_{p=1}^{l} \lambda_{i j}^{k p} r_{p}+r_{i j}=-\left(p_{i j}^{k}-f(x) q_{i j}^{k}\right), \\
(i, j) \in U^{*}, k \in K_{S}^{0}(i, j) ; \\
u_{i}^{k}-\mu_{i j}^{k} u_{j}^{k}+\sum_{p=1}^{l} \lambda_{i j}^{k p} r_{p}=-\left(p_{i j}^{k}-f(x) q_{i j}^{k}\right), \\
(i, j) \in U \backslash U^{*}, k \in K_{S}(i, j) \text { or }(i, j) \in U^{*}, k \in K_{S}(i, j) \backslash K_{S}^{0}(i, j) .
\end{gathered}
$$

Consider the effective algorithm for solving sparse system of potentials which is based on principles of decomposition of sparse system (11)-(13).

Let's construct a matrix

$$
\begin{gathered}
D=\left(\begin{array}{c}
D_{1} \\
D_{2}
\end{array}\right), D_{1}=\left(\Lambda_{\tau \rho}^{k p}, p=\overline{1, l}, t(\tau, \rho)^{k}=\overline{1,\left|U_{B}\right|}\right), \\
D_{2}=\left(\delta_{i j}\left(B_{\tau \rho}^{k}\right), \xi(i, j)=\overline{1,\left|U^{*}\right|}, t(\tau, \rho)^{k}=\overline{1,\left|U_{B}\right|}\right),
\end{gathered}
$$

$\xi=\xi(i, j)-$ a number of $\operatorname{arc}(i, j) \in U^{*}, \xi \in\left\{1,2, \ldots,\left|U^{*}\right|\right\}$, where

$$
\Lambda_{\tau \rho}^{k p}=\lambda_{\tau \rho}^{k p}+\sum_{(i, j)^{k} \in U_{L}^{k}} \lambda_{i j}^{k p} \delta_{i j}^{k}(\tau, \rho),(\tau, \rho)^{k} \in U^{k} \backslash U_{L}^{k} .
$$

$\delta^{k}(\tau, \rho)=\left(\delta_{i j}^{k}(\tau, \rho), \quad(i, j)^{k} \in U^{k}\right)$ - characteristic vector, entailed by arc $(\tau, \rho)^{k} \in U^{k} \backslash U_{L}^{k}$ concerning a support $U_{L}^{k}$ for system (2), $k \in K, \widetilde{t}=\left|U_{B}\right|$, 
$U_{B}=\left\{U_{B}^{k}, k \in K\right\}[2,4]$.

The vector

$$
r=\left(r_{p}, p=\overline{1, l} ; r_{i j},(i, j) \in U^{*}\right),
$$

we compute from the system

$$
D^{\prime} r=\omega
$$

where $\omega=\left(\omega_{t}, t=\overline{1, \widetilde{t}}\right)$,

$$
\omega_{t}=-\sum_{(i, j)^{k} \in B_{\tau \rho}^{k}}\left(p_{i j}^{k}-f(x) q_{i j}^{k}\right) \delta_{i j}^{k}(\tau, \rho), t=t(\tau, \rho)^{k}, k \in K
$$

The system (14) has unique solution, as $\operatorname{det} D \neq 0$ [4].

For each $k \in K$ let us put $u_{i}^{k}=0$ for some $i \in I^{k}$. The other components of vectors $u^{k}=\left(u_{i}^{k}: i \in I^{k}\right), k \in K$ are uniquely determined by the system (15):

$$
\begin{gathered}
u_{i}^{k}-\mu_{i j}^{k} u_{j}^{k}=-\sum_{p=1}^{l} \lambda_{i j}^{k p} r_{p}-\left(p_{i j}^{k}-f(x) q_{i j}^{k}\right), \\
(i, j) \in U \backslash U^{*}, k \in K_{S}(i, j),(i, j)^{k} \in U_{L}^{k} ; \\
u_{i}^{k}-\mu_{i j}^{k} u_{j}^{k}=-\sum_{p=1}^{l} \lambda_{i j}^{k p} r_{p}-r_{i j}-\left(p_{i j}^{k}-f(x) q_{i j}^{k}\right), \\
(i, j) \in U^{*}, k \in K_{S}^{0}(i, j),(i, j)^{k} \in U_{L}^{k} ; \\
u_{i}^{k}-\mu_{i j}^{k} u_{j}^{k}=-\sum_{p=1}^{l} \lambda_{i j}^{k p} r_{p}-\left(p_{i j}^{k}-f(x) q_{i j}^{k}\right), \\
(i, j) \in U^{*}, k \in K_{S}(i, j) \backslash K_{S}^{0}(i, j),(i, j)^{k} \in U_{L}^{k} .
\end{gathered}
$$

The system (15) consists from $|K|$ independent subsystems. For calculation nonzero components of vector $u^{k}=\left(u_{i}^{k}: i \in I^{k}\right)$ for every independent subsystem (15) for fixed $k \in K$ we are able to take using $O\left(\left|I^{k}\right|\right)$ arithmetical operations, where $\left|I^{k}\right|$ - the number of nodes of the graph $G^{k}=\left(I^{k}, U^{k}\right)$. The algorithm with $O(n)$ computational complexity in the worst case is used for calculation nonzero component of everyone characteristic vector $\delta^{k}(\tau, \rho)$, where $n=\left|I^{k}\right|[2,4]$.

We add to a vector $r=\left(r_{p}: p=\overline{1, l} ; r_{i j},(i, j) \in U^{*}\right)$ the following components $r_{i j}=0,(i, j) \in U_{0} \backslash U^{*}$. Let's receive a new vector

$$
\widetilde{r}=\left(r_{p}: p=\overline{1, l} ; r_{i j},(i, j) \in U^{*} ; r_{i j}=0,(i, j) \in U_{0} \backslash U^{*}\right)
$$


A reduced costs $\widetilde{\Delta}_{i j}^{k}$ we calculate for the $\operatorname{arcs}(i, j)^{k} \in U_{N}^{k}, U_{N}^{k}=U^{k} \backslash U_{S}^{k}$, $k \in K$ and also for the $\operatorname{arcs}(i, j)^{k}, k \in K_{S}^{0}(i, j),(i, j) \in U^{*}$, using the formulas:

$$
\widetilde{\Delta}_{i j}^{k}=-\left(p_{i j}^{k}-f(x) q_{i j}^{k}\right)-\left(u_{i}^{k}-\mu_{i j}^{k} u_{j}^{k}+\sum_{p=1}^{l} \lambda_{i j}^{k p} r_{p}\right) .
$$

\section{References}

[1] R. Gabasov, F.M. Kirillova, Methods of Linear Programming. Part 3: Special Problems, Minsk, BSU (1980), In Russian.

[2] L.A. Pilipchuk, Linejnyje Neodnorodnyje Zadachi Potokovogo Programmirovanija, Minsk, BSU (2009), In Russian.

[3] L.A. Pilipchuk, E.S. Vecharynski, Y.H. Pesheva, Solution of large linear systems with embedded network structure for a non-homogeneous network flow programming problem, Mathematica Balkanica, 22, Fasc. 3-4, (2008), $235-254$.

[4] L.A. Pilipchuk, Razrejennyje Nedoopredelennyje Sistemi Lineinix Algebraicheskix Uravnenii, Minsk, BSU (2012), In Russian. 
